\title{
Perfis de Personalidade de Adolescentes que Cometeram Homicídio
}

\author{
Carolina Cardoso de Soura - Pontifícia Universidade Católica de Goiás, Goiânia, Brasil \\ Ana Cristina Resende - Pontifícia Universidade Católica de Goiás, Goiânia, Brasil
}

\begin{abstract}
Resumo
O objetivo deste presente trabalho foi caracterizar perfis psicológicos em um grupo de adolescentes que cometeram homicídio. Participaram do estudo 33 adolescentes, do sexo masculino e feminino, com idades entre 14 e 19 anos, que cometeram homicídio e estão cumprindo medidas socioeducativas. Foram utilizados como instrumentos uma entrevista semiestruturada e o método de Rorschach (Sistema Compreensivo). Os adolescentes revelaram características que permitiram compreendê-los em três subgrupos: um grupo com componentes narcísicos patológicos mais frequentes, outro grupo que se destaca pela falta de habilidades sociais e interpessoais, com tendência a estabelecer relacionamentos conflituosos e com pouca capacidade de lidar com as demandas da vida diárias, e o último grupo apresentou juízo crítico e coerência rebaixados, como pensamentos excêntricos e desconsideração pelos comportamentos socialmente esperados. Os principais resultados permitiram constatar a insuficiência de recursos psicológicos que interferem na capacidade de adaptação ao meio em que vivem.

Palavras-chave: adolescentes, homicídio, método de Rorschach
\end{abstract}

Profiles of Personality of Adolescents who have Committed Homicide

\begin{abstract}
This study consists of an empirical article that aims to characterize psychological profiles in a group of adolescents who committed homicide. The study included 33 adolescents, male and female, aged between 14 and 19 years, who committed homicide and are serving educational measures deprived of freedom. The teenagers were submitted to a semi directed interview and the Rorschach Test (Comprehensive System). The results showed some personality traits that allowed them to be divided into three subgroups: a group with marked narcissistic pathological components; another group that is characterized by absence of social and interpersonal skills, with a tendency to establish conflictive relationships and with low capacity to cope with the demands of daily life; and the latter group had demoted critical judgment and consistency as eccentric thoughts and disregard for the socially expected behaviors. The main results demonstrated the insufficiency of psychological resources that interfere with their capacity to adapt to the environment.

Keywords: adolescents, homicide, Rorschach test
\end{abstract}

\section{Perfiles de Personalidad de Adolescentes que Cometieron Homicidios}

\begin{abstract}
Resumen
El objetivo de este estudio fue diferenciar perfiles psicológicos en un grupo de 33 adolescentes que cometieron homicidio, de ambos sexos, con edades entre 14 y 19 años, y que están cumpliendo medidas socioeducativas. Una entrevista semiestructurada y el método de Rorschach (Sistema Comprensivo) fueron utilizados como instrumentos. Los adolescentes revelaron algunas características que permitieron comprenderlos en tres sub-grupos: un grupo con componentes narcisistas patológicos más frecuentes; otro grupo que se caracteriza por la falta de habilidades sociales e interpersonales, con una tendencia a establecer relacionamientos conflictivos y con poca capacidad para hacer frente a las exigencias de la vida diaria; y el último grupo presentó juicio crítico y coherencia disminuidos, como pensamientos excéntricos y desconsideración por los comportamientos socialmente esperados. Los principales resultados constataron insuficiencia de recursos psicológicos que interfieren en la capacidad de adaptarse al medio en que viven.

Palabras clave: adolescentes, homicidio, método de Rorschach
\end{abstract}

O interesse científico em estudos com adolescentes que se envolveram em atos infracionais tem avançado especialmente nos últimos 10 anos. O aumento da violência praticada por esses jovens traz temores e mobiliza a população que, por sua vez, exige medidas de repressão, deixando de lado o conhecimento das razões dessa crescente violência, dos fatores que motivam esse tipo de comportamento, as formas de atenção e prevenção necessárias para o enfrentamento da questão. A discussão da maioridade penal é um dos exemplos dessa mobilização (Assis, 1999; Paludo, 2011; Minayo \& Souza, 2003).

Segundo Gauer, Davoglio e Vasconcelos (2012), é importante conhecer a realidade de adolescentes que cometeram atos violentos, mais especificamente o homicídio, o latrocínio e o estupro, distinguindo daqueles que cometeram atos não violentos, tais como furto, roubo, tráfico e receptação. A violência não se manifesta isoladamente, é o resultado da complexa interação de fatores de risco (biológicos; familiares, tais como ser 
filho de pais condenados ou que abusam fisicamente e psicologicamente dos filhos; situacionais, tais como baixo rendimento escolar ou fácil acesso às armas e às drogas psicoativas; sociais e individuais, como comportamentos e características de personalidade) por certo período de tempo, especialmente durante a infância, que encoraja o indivíduo ao comportamento violento (Assis, Avanci, Santos, Malaquias, \& Oliveira, 2004; Bartol, 2008; Freitas \& Ramires, 2011; Gallo \& Williams, 2005; Gomide; 2010; Kodato e Silva, 2000; OMS, 2002; Roe-sepowitz, 2009).

Considerando a personalidade como um dos fatores que pode influenciar no comportamento violento de adolescentes, muitos estudos têm investigado como essas características estão relacionadas com a conduta criminosa em adolescentes. Por exemplo, quando se trata de desistência ou de reincidência da conduta infracional algumas características de personalidade têm-se destacado. A autoestima positiva, o autocontrole, a responsabilidade, a competência, a capacidade de tomar decisões, a flexibilidade e o temperamento afetuoso são aspectos considerados facilitadores do processo de desistência do crime (Costa \& Assis, 2006). Por outro lado, o déficit em elementos da maturação psicossocial, especialmente no controle de impulsos e na supressão da agressividade, está negativamente relacionado à desistência dessa conduta (Monahan, Steinberg \& Cauffman, 2009; Ayer, Williams, Tolan, \& Wilson, 2013).

Alguns estudos de metanálise com adolescentes que cometeram homicídio têm apontado os problemas cognitivos, especialmente os distúrbios da função executiva, como preditores de seus crimes (Busch, et al., 2009; Busch, Zagar, Hughes, Arbit, \& Bussell, 1990; Zagar, Busch, Grove, Hughes, \& Arbit, 2009). Vries e Liem (2011), em uma investigação com base em padrões de comportamentos, pesquisaram 137 jovens que cometeram homicídio e concluíram que a reincidência nesse tipo de crime é mais comum em adolescentes do sexo masculinos com capacidade de autocontrole prejudicada.

Outros estudos apontam que os adolescentes que cometeram ato infracional violento tendem a apresentar traços antissociais mais marcantes, do que os autores de outros atos infracionais, tais como falta de interesse pelo outro, ausência de ansiedade, culpa e remorso (Souza \& Resende, 2012a; 2012b; Resende, 2011; Souza \& Resende, 2011; Dicataldo \& Everett, 2008; Schmitt, Pinto, Gomes, Quevedo, \& Stein, 2006). $\mathrm{O}$ traço antissocial tem sido empregado para designar o caráter agressivo e desafiador da conduta de indivíduos que apresentam comportamentos inadequados que causam prejuízos no seu funcionamento social, além de aumentar as chances de desenvolver traços psicopáticos no futuro (Pacheco, Alvarenga, Reppold, Piccinini, \& Hutz, 2005; Schmitt et al., 2006; Viljoen, MacDougall, Gagnon, \& Douglas, 2010).

As avaliações dos jovens que cometeram atos infracionais por meio do Modelo dos Cinco Grandes Fatores Personalidade, proposto por Goldberg (1992), apontam que a dimensão Socialização, relacionada à orientação pró-social com as pessoas, à confiança e ao altruísmo, tende a ser um traço pouco encontrado (Corff \& Toupin, 2009; Dam, Janssens, \& De Bruyn, 2005). Pontuações baixas em Socialização costumam ser comuns em pessoas cínicas, manipuladoras, vingativas, antagonistas, autocentradas, indiferentes para com as necessidades alheias, hostis e com tendência a comportamentos antissociais (Howard \& Howard, 1995; Hutz et al., 1998).

Outros dois traços têm sido observados em condutas antissociais de jovens que cometeram crimes violentos. O traço Busca de sensações, caracterizado por uma tendência a procurar novas e variadas experiências e sensações, uma disposição para correr riscos com a finalidade de satisfação (Formiga, Aguiar, \& Omar, 2008; Romero, Luengo \& Sobral, 2001); e o traço insensibilidade (callous-unemotion) caracterizado pela ausência de emoções nas relações interpessoais (Frick \& White, 2008; Kimonis, Frick, Munoz, \& Aucoin, 2007; McMahon, Witkiewitz, \& Kotler, 2010).

Estudos com o método de Rorschach Sistema Compreensivo (SC) também têm discriminado, em termos de características de personalidade, adolescentes mais propensos aos comportamentos violentos, do que aqueles bem menos propensos a se comportar dessa maneira. Mais especificamente, as investigações com jovens que cometeram homicídio têm destacado várias características no âmbito das interações interpessoais, autopercepção, afetos e aspectos cognitivos. Quando se trata das interações interpessoais e recursos eficientes para lidar com situações estressantes (coping), prevalecem a predisposição para gerar conflitos nos seus relacionamentos (PHR > GHR), a pouca disponibilidade para a cooperação e solicitude com o outro (COP $\downarrow$ ), o desinteresse pelas pessoas, pelo que elas fazem ou dizem $(\mathrm{H} \downarrow)$ e a falta de habilidades sociais (EA $\downarrow$ ) (Corredor, 2000; Durat, 2006; Exner \& Sendín, 1999; Gacono, Gacono \& Evans, 2008; Gacono \& Meloy, 1994; Garcia, 1999; Loving \& Russell, 2000; Lowen, 1983; Olin \& Keatinge, 
1998; Resende, 2009; 2011; Resende \& Souza, 2011; Souza \& Resende, 2012b). Para esses jovens é indicado tratamento com foco na redução da ansiedade interpessoal e no treinamento de habilidades sociais, com o objetivo de aumentar a autoestima e a qualidade relacional (Del Prette \& Del Prette, 2001).

Com relação à autopercepção, nota-se a ausência de um senso estável de quem o jovem realmente é, a dificuldade de se identificar com pessoas reais e que fazem parte do seu cotidiano $(\mathrm{H}<(\mathrm{H})+(\mathrm{Hd})+\mathrm{Hd})$, bem como a presença frequente de traços narcísicos, típico de pessoas egoístas que tendem a culpar os outros por seus erros e fracassos $(\mathrm{Fr}+\mathrm{rF}>0)$, e também a baixa autoestima (Ego $\downarrow)$. Em relação aos aspectos afetivos, destacam-se a ausência de culpa e remorso por atitudes e decisões mal tomadas [Somb $\downarrow($ SumV $=0)$ ], a capacidade insuficiente de reconhecer e expressar os seus sentimentos (WSumC $\downarrow$ ), a pouca capacidade de lidar com situações emocionalmente complexas (Blends $\downarrow$ ) e a menor disposição para participar de situações que envolvem expressões de afetos (Afr $\downarrow$ ) (Corredor, 2000; Durat, 2006; Gacono \& Meloy,1994; Gacono et al., 2008; Garcia, 1999; Loving \& Russell, 2000; Lowen, 1983; Olin \& Keatinge, 1998; Resende, 2011; Resende \& Souza, 2011; Souza \& Resende, 2012b).

Além dessas características, frequentemente são citadas as limitações no aspecto cognitivo, tais como rebaixamento de juízo crítico e da coerência $(\mathrm{F}+\% \downarrow)$, a desconsideração pelos comportamentos socialmente esperados $(\mathrm{Xu} \% \uparrow)$, a tendência a interpretar o que as pessoas fazem e falam de modo equivocado (M- $\uparrow$ ), bem como a falta de motivação para captar e organizar as informações de modo criativo e eficiente $(\mathrm{Z} \downarrow \downarrow)$, revelando superficialidade, um exame rápido e pouco cuidadoso das circunstâncias, o que leva esse jovem a cometer mais erros e a tomar decisões precipitadas. Quando se trata de jovens homicidas que atuam de forma repentina e sem pensar nas consequências de seus atos, as características no Rorschach mais evidenciadas têm sido a pouca capacidade de lidar com situações emocionalmente complexas, precipitando-se na tomada de decisões e estressando-se facilmente quando está diante dessas circunstâncias que não foram planejadas (Blends $\downarrow$; L $\uparrow$ ) (Durat, 2006; Gacono et al., 2008; Gacono \& Meloy, 1994; Olin \& Keatinge, 1998; Resende, 2011; Resende \& Souza, 2011; Souza \& Resende, 2012b). Grande parte desses jovens apresenta dificuldades para gerenciar situações básicas da vida diária sem a ajuda ou a supervisão de um profissional (Souza \& Resende, 2012b).
Nas investigações de Gacono et al. (2008), Gacono e Meloy (1994) e Loving e Russell (2000), os autores observaram também as dificuldades em lidar com sentimentos de raiva, demonstrando comportamento opositor excessivo ( $\mathrm{S} \uparrow$ ), e expressão da agressão de modo mais primitivo, muitas vezes exercida no dia a dia como uma conduta natural de resolução de problemas e, portanto, pouco consciente do seu papel nocivo nas relações $(\mathrm{AgC})$. Os autores salientaram ainda que a integração de algumas características de personalidade podem gerar comportamentos bastante perniciosos, tal como a exploração e manipulação do outro para atingir seus próprios fins, sem qualquer indício de remorso ou arrependimento $(\mathrm{Fr}+\mathrm{rF}>0+\mathrm{SumV}=0+\mathrm{H}=0+$ COP $=0$ ). Borges e Alencar (2006) e Manita (2000) apontam quando há arrependimento no cometimento de atos infracionais, geralmente está relacionado à interpretação do ato como acidental ou quando há uma consequência punitiva pós-crime. $\mathrm{O}$ tratamento indicado para esses adolescentes seria uma psicoterapia em grupo, com imposição de limites, com outras pessoas que apresentam as mesmas características para que possam começar a identificar suas próprias características um no outro (Costa \& Valerio, 2008; Gabbard, 1998; Kaplan \& Sadock, 2007).

De uma forma geral, as pesquisas apontam que a avaliação de personalidade desses jovens possibilita uma diferenciação mais precisa de quanto a atividade infracional remete a déficits no desenvolvimento, grau de envolvimento com a prática criminosa, natureza e tempo de intervenção requerido, nível de adaptação aos contextos institucionais, se há algum tipo de transtorno e a repercussão disso na vida do adolescente, quais as características de personalidade dominantes, assim como permite prever uma maior ou menor probabilidade de reincidir no crime (Caldwell, McCormick, Umstead, \& Van Rybroek, 2007; Dam et al., 2005; Frick \& White, 2008; Ridenour, Marchant, \& Dean, 2001; Salekin, 2002, Kimonis, et al., 2007). Diante do exposto, entende-se que esses jovens constituem um grupo heterogêneo de pessoas e o presente estudo tem o objetivo de explorar as possíveis configurações psicológicas, em termos de características de personalidade mais dominantes, em um grupo de adolescentes que cometeram homicídio e estão cumprindo medidas socioeducativas. 


\section{Método}

\section{Participantes}

Participaram do estudo 33 adolescentes, sendo oito $(24,2 \%)$ do sexo feminino e $25(75,8 \%)$ do sexo masculino, com idades entre 14 e 19 anos, escolaridade variada entre ensino fundamental e ensino médio que cometeram homicídio ou latrocínio (roubo seguido de morte) e estavam cumprindo medida socioeducativa de internação.

O critério de exclusão foi desempenho insuficiente no teste para fornecer informações confiáveis e para sustentar interpretações válidas (número de respostas menor do que 14 e/ou com rejeição de cartões). Todos os adolescentes convidados concordaram em participar deste estudo e apresentaram os critérios para serem incluídos. Não houve necessidade de aplicação do critério de exclusão.

\section{Instrumentos}

Entrevista Semiestruturada com os Adolescentes: com a finalidade de estabelecer um rapport e recolher informações breves acerca de características de identificação, capacidade de comunicação e orientação alopsíquica e autopsíquica, histórico do envolvimento em atos infracionais.

Prontuários dos Adolescentes: utilizado para levantar informações sobre os adolescentes durante o cumprimento da medida socioeducativa, incluindo o tempo de internação, o(s) ato(s) infracional(nais) cometido(s) e para confirmar informações colhidas na entrevista.

Método de Rorschach (Sistema Compreensivo): é composto por dez cartões com manchas de tinta, os quais servem de estímulos pouco organizados e levam o indivíduo em avaliação a expressar conteúdos associativo-perceptivos representativos de sua forma habitual de pensar, sentir e agir. É administrado individualmente e está mais intimamente relacionado com os comportamentos que as pessoas utilizam para resolver os seus problemas do que associado ao que elas falam. Assim, a personalidade é revelada por meio da ação de solucionar um problema. Identificar o que cada mancha de tinta pode ser e, posteriormente, descrever o que na mancha fez parecer o que foi descrito. Cada resposta, ou solução para a tarefa, é codificada de acordo com orientações padronizadas e, por meio de dimensões ou códigos, são então resumidas em escores e interpretados no Sistema Compreensivo (Exner, 2003).
Para essa investigação, foram consideradas apenas as variáveis relacionadas na Tabela 1 , levantadas em outros estudos com adolescentes que cometeram atos infracionais e utilizaram o Método de Rorschach como instrumento de coleta de dados (Corredor, 2000; Durat, 2006; Gacono \& Meloy, 1994; Gacono et al., 2008; Garcia, 1999; Loving \& Russell, 2000; Lowen, 1983; Olin \& Keatinge, 1998; Resende, 2011; Resende \& Souza, 2011; Souza \& Resende, 2012b). Algumas variáveis citadas pelos autores Gacono e Meloy (1994), Gacono et al., (2008) e Souza e Resende (2012b) foram classificadas como mais típicas de características antissociais nos adolescentes, também chamadas de indicadores de Transtorno de Conduta. Essas variáveis estão relacionadas a alguns aspectos da autopercepção e da configuração dos afetos e emoções e foram agrupadas em um subitem chamado Características de Psicopatia, conforme Tabela 1. As demais variáveis explicitadas pelos autores não entraram no grupo de Características de Psicopatia, mas puderam ser agrupadas em dois subitens (Déficits Cognitivos e Déficits Relacionais) conforme agrupamentos comuns de códigos (clusters) propostos por Exner (2003), utilizados para a interpretação habitual dos dados do Rorschach. Assim, as demais variáveis agrupadas em Déficits Relacionais consistem em índices que compõem o Índice de Déficits Relacionais, o CDI, o índice de imaturidade para lidar com questões emocionalmente complexas (Blends) e o índice indica baixa autoestima $(\mathrm{Ego}<0,37)$, que sugere falta de autoconfiança e predisposição para ser influenciável por outras pessoas. Já as variáveis que compõem o agrupamento de Déficits Cognitivos são todas explicitadas nos clusters de índices referentes aos aspectos cognitivos do teste (processamento, mediação e ideação) (Exner, 2003; Exner \& Sendín, 1999; Resende, 2009).

\section{Procedimentos}

O projeto foi submetido e aprovado pelo Juizado da Infância e Juventude do Estado e posteriormente pelo Comitê de Ética. A participação dos sujeitos na pesquisa foi voluntária e autorizada por meio do Termo de Consentimento Livre e Esclarecido pelos próprios adolescentes.

Após essa fase, as instituições socioeducativas foram contatadas para levantamento do quantitativo de adolescentes que cometeram homicídio e agendamento da coleta de dados. No recrutamento, todos os participantes foram convidados pessoalmente e estimulados a participar do estudo. 
Tabela 1

Variáveis do Rorschach e seu Significado no Agrupamento das Diferentes Características de Personalidade

\begin{tabular}{|c|c|c|}
\hline $\begin{array}{l}{ }^{1} \text { Características } \\
\text { de Personalidade }\end{array}$ & $\begin{array}{l}{ }^{2} \text { Variável do } \\
\text { Rorschach }\end{array}$ & Significado \\
\hline \multirow{9}{*}{$\begin{array}{l}\text { Déficits } \\
\text { cognitivos }\end{array}$} & $\mathrm{XA} \%<0,70$ & Baixo índice geral de ajustamento da realidade. \\
\hline & $\begin{array}{l}\text { WDA } \% \\
<0,75\end{array}$ & $\begin{array}{l}\text { Rebaixamento do índice mais preciso de ajustamento indicando pouca } \\
\text { capacidade de perceber a realidade mais convencional e óbvia. }\end{array}$ \\
\hline & $\mathrm{Xu} \% \geq 0,38$ & Desconsideração pelos comportamentos socialmente esperados. \\
\hline & $\mathrm{X}-\% \geq 0,40$ & Desajustamento da realidade. \\
\hline & $\mathrm{P} \leq 2$ & $\begin{array}{l}\text { Repostas populares abaixo da média revelam pouca adaptação às normas de } \\
\text { convivência em grupo. }\end{array}$ \\
\hline & PTI $\geq 2$ & Indica a presença de distúrbios cognitivos \\
\hline & M- $>1$ & $\begin{array}{l}\text { A presença de movimentos humanos de qualidade formal menos (FQ-) aponta } \\
\text { tendência a interpretar o que as pessoas fazem e falam de modo equivocado. }\end{array}$ \\
\hline & Zf $\leq 4$ & Baixa frequência de nota $Z$ assinala capacidade cognitiva rebaixada. \\
\hline & $\mathrm{DQ}+<4$ & $\begin{array}{l}\text { Poucas respostas de qualidade evolutiva sintetizada demonstram nível } \\
\text { intelectual pouco elaborado. }\end{array}$ \\
\hline \multirow[t]{7}{*}{$\begin{array}{l}\text { Déficits de } \\
\text { relacionais }\end{array}$} & $\mathrm{CDI} \geq 5$ & $\begin{array}{l}\text { Índice de déficit nos relacionamentos interpessoais, este índice quando } \geq 5 \\
\text { indica predisposição para desorganização funcional nos relacionamentos ou na } \\
\text { administração do estresse. }\end{array}$ \\
\hline & $\mathrm{COP}=0$ & $\begin{array}{l}\text { Movimento cooperativo igual a zero indica pouca disponibilidade para a } \\
\text { cooperação com o outro. }\end{array}$ \\
\hline & PHR $>$ GHR & $\begin{array}{l}\text { Índice de representação humana pobre maior do que o índice de representação } \\
\text { humana boa aponta para a disponibilidade para relacionamentos conflituosos e } \\
\text { comportamentos sociais inadequados. }\end{array}$ \\
\hline & $\mathrm{EA} \leq 3$ & Experiência efetiva indica poucos recursos para lidar com situações diárias. \\
\hline & Blends $\leq 2$ & $\begin{array}{l}\text { Poucas respostas com determinantes mistos indicam escassa capacidade de } \\
\text { lidar com situações emocionalmente complexas. }\end{array}$ \\
\hline & $\mathrm{L} \geq 2$ & Lambda alto aponta para um estilo evitativo de enfrentamento das situações. \\
\hline & Ego $<0,37$ & Índice de egocentrismo rebaixado demonstra baixa autoestima. \\
\hline \multirow[t]{7}{*}{$\begin{array}{l}\text { Características } \\
\text { de psicopatia }\end{array}$} & $\mathrm{Fr}+\mathrm{rF}>0$ & $\begin{array}{l}\text { Pode revelar narcisismo pernicioso, típico de pessoas com uma determinação } \\
\text { hostil em explorar e manipular o outro para atingir seus próprios fins. }\end{array}$ \\
\hline & H puro $\leq 1$ & Poucos conteúdos humanos inteiros evidencia falta de interesse pelas pessoas \\
\hline & SumV $=0$ & A ausência de sombreado vista aponta a falta de culpa e remorso. \\
\hline & SumSomb $\leq 1$ & Baixo somatório de sombreados demonstra ausência de ansiedade. \\
\hline & $\begin{array}{l}\text { WSumC } \leq \\
1,5\end{array}$ & $\begin{array}{l}\text { Soma ponderada das respostas cromáticas rebaixada assinala capacidade } \\
\text { insuficiente de reconhecer e expressar os seus sentimentos. }\end{array}$ \\
\hline & Afr $<0,40$ & $\begin{array}{l}\text { Índice de afetividade abaixo da média revela menos disposição para participar } \\
\text { de situações que envolvem expressões de afetos. }\end{array}$ \\
\hline & Sum $\mathrm{T}=0$ & $\begin{array}{l}\text { A ausência de textura, associada à variável } \mathrm{H} \text { puro } \leq 1 \text { fortalece o argumento } \\
\text { de falta de interesse por relacionamentos mais próximos. }\end{array}$ \\
\hline
\end{tabular}

${ }^{1}$ O grupo Características de psicopatia foi baseado nos próprios estudos acima, que trouxeram essa denominação para esse grupo de variáveis. Os demais grupos: Déficits relacionais e Déficits cognitivos foram criados utilizando as variáveis apresentadas pelos estudos acima e de acordo com os agrupamentos de variáveis do teste proposto por Exner (2003).

${ }^{2}$ Todas as variáveis foram levantadas em estudos com adolescentes que cometeram atos infracionais ou que foram diagnosticados com Transtorno de Conduta e Transtorno de Personalidade Antissocial: Corredor (2000), Durat (2006), Gacono e Meloy (1994), Gacono et al. (2008), Garcia (1999), Loving e Russell (2000), Lowen (1983), Olin e Keatinge (1998), Resende (2011); Resende e Souza (2011), Souza e Resende (2012b). 
A coleta de dados foi realizada de forma individual, em dois encontros de aproximadamente 1 hora e 30 minutos cada, numa sala adequada para atendimento e testagem psicológica, nas próprias instituições, onde regularmente as psicólogas realizavam seus atendimentos. No primeiro encontro, a entrevista semiestruturada foi administrada $(N=28)$. Em outro encontro, era realizada a aplicação do Método de Rorschach $(N=33)$. Cinco adolescentes não foram submetidos à entrevista porque estavam finalizando sua sanção socioeducativa e não haveria tempo hábil para a realização dos dois instrumentos.

No que diz respeito aos procedimentos de análise dos dados, o primeiro passo consistiu em caracterizar a amostra. Quanto aos protocolos de Rorschach, foram codificados às cegas e separadamente por dois juízes experientes no Sistema Compreensivo. Foi realizada a análise de concordância entre os codificadores por meio do Kappa. Os resultados observados indicam que houve boa concordância entre os juízes ( $>$ $0,76)$, com exceção dos códigos especiais cujo índice foi limítrofe $(0,65)$. De uma forma geral, a correção do Método de Rorschach para este estudo foi considerada confiável.

Considerando a análise dos dados do Rorschach em primeiro lugar, observou-se a possibilidade de os distúrbios cognitivos serem os responsáveis pelos comportamentos socialmente desadaptados. Quando três ou mais variáveis estivessem presentes nesse grupo de variáveis (Déficits Cognitivos), o adolescente seria classificado tendo esse aspecto como o mais prejudicado em sua personalidade. Logo em seguida, foram priorizados os Déficits Relacionais, observando também a frequência de três ou mais índices presentes no protocolo do adolescente. Por último foram consideradas as Características de Psicopatia quando quatro ou mais variáveis estivessem presentes no protocolo. Essa estratégia de analisar os dados foi baseada na estratégia de interpretação do Sistema Compreensivo (Exner, 2003), em que os distúrbios do pensamento são considerados inicialmente como a primeira variável-chave para a interpretação dos dados (p. 228). Logo em seguida estão as variáveis relacionadas à depressão (DEPI) e aos déficits relacionais (CDI). Como as questões referentes à depressão não constaram entre os índices mais frequente em adolescentes que cometeram homicídio ou que cometem atos infracionais, privilegiou-se as variáveis que indicam os déficits relacionais.

\section{Resultado e Discussão}

A idade média dos 33 participantes foi 16,85 anos $(D P=1,09)$, sendo a maioria com 17 anos $(N=10$, $35 \%)$. A média de anos de estudo foi de 7,64 (DP = 2,37 ), dado que corrobora com a literatura, a qual evidencia que o abandono ou o baixo rendimento escolar é um importante fator de risco associado ao envolvimento de jovens envolvidos com atos infracionais (Gallo \& Williams, 2005; Kodato e Silva, 2000).

Boa parte dos adolescentes $(46,4 \%, N=13)$ relatou ter planejado e realizado com frieza o homicídio. Houve relato de arrependimento em doze casos (43\%). No caso desses adolescentes, a verbalização do arrependimento vem acompanhada da consequência que teria ocasionado o ato: o fim da liberdade. Observa-se, portanto, que muitos adolescentes deste estudo $(N=$ 16, 57\%) não demonstraram sentimento de culpa ou remorso pelo ato praticado.

De acordo com estudo realizado por Borges e Alencar (2006) com adolescentes que cometeram homicídio, a mudança ocorrida na forma de pensar dos agressores parece ter sido determinada pelas consequências adversas sofridas após a transgressão e não pelo ato em si. Isso indica que a maioria responderia que atualmente mataria outra vez diante das mesmas circunstâncias e julgaria como correto o homicídio cometido. Todos os adolescentes que cometeram latrocínio $(N=3,10,7 \%)$ se declararam arrependidos. Justificaram que a vítima reagiu, mas que a intenção não era o homicídio, e sim o roubo. Ou seja, no caso desses jovens, o arrependimento estava relacionado ao fato de tirar a vida de outros, e não ao assalto.

Dos outros seis adolescentes $(21,5 \%)$ que declararam arrependimento, dois $(7,2 \%)$ relataram ter assassinado a mãe e a namorada por acidente, por efeito do uso de drogas. Um deles declarou ter tido alucinações auditivas que o induziram a cometer o ato contra a mãe e o outro revelou que, devido ao efeito da droga, não dimensionou a força que colocava na namorada em uma brincadeira de luta. A associação do ato ao crime, de acordo com Manita (2000), é designada como uma "recusa de atribuição de significado" nomeada como "acidente", e traduz as situações ditas como impensadas e incontroladas. Segundo a autora, essa dimensão de significado tem relevância no caso dos primeiros atos infracionais e não se aplica em casos de reincidentes no crime. Esse foi o caso dos adolescentes deste estudo, que apresentaram essa 
justificativa para o ato e não haviam cometido atos infracionais anteriores a esse.

Os quatro últimos adolescentes $(14,3 \%)$ que relataram arrependimento foram motivados a cometer o ato por brigas com pessoas próximas, colegas de escola, irmão e, no caso de uma adolescente, uma briga com o esposo resultou no homicídio contra o filho, por vingança. No caso desses adolescentes, algumas características individuais podem justificar o ato, como pouca capacidade de lidar com situações emocionalmente complexas, precipitando-se na tomada de decisões e estressando-se facilmente quando se está diante dessas circunstâncias, ou tendência a interpretar o que as pessoas fazem e falam de modo equivocado e dificuldade de controlar os afetos, com tendência a violentas descargas emocionais (Gacono et al., 2008; Gacono \& Meloy, 1994).

Os adolescentes que não relataram arrependimento, 14 (50\%) foram motivados por dívidas do tráfico e brigas com inimigos de gangue, brigas em festas com pessoas desconhecidas e disputa por namoradas. No caso desses adolescentes, o não arrependimento do ato pode estar vinculado ao que Paludo (2011) chama de autoafirmação, quando a transgressão, o comportamento violento e infracional fazem parte do processo de visibilidade social. Assim, a busca pelo respeito se dá por meio de atos que os excluem da sociedade, uma vez que assumindo o papel de perigosos passarão a ser notados, mesmo que seja pelo medo que incitam nas pessoas.

As outras duas adolescentes $(N=2 ; 7,2 \%)$ que não demonstraram arrependimento foram motivadas por brigas familiares e as vítimas foram o pai e a avó. No caso da adolescente responsável pelo homicídio da avó, este foi confirmado pela perícia criminal. Ela nega o ato, mas relata que ela e a avó brigavam com frequência, especialmente devido ao seu envolvimento com o tráfico.

Já no caso da adolescente que matou o pai, sua motivação corrobora com os estudos de Roe-sepowitz (2009) e Gomide (2010). A jovem revelou que o pai era alcoólatra e que ela sofria abusos físicos diariamente. Segundo os autores, adolescentes que cometem parricídio comumente apresentam como motivação terem sido vítimas de abusos pelo pai.

Ao relacionar os adolescentes que não se mostraram arrependidos com as características de personalidade que eles apresentam, os resultados demonstram que esses adolescentes não constituem como um grupo homogêneo quanto à personalidade.
Sete $(44 \%)$ do total de adolescentes $(N=16,57 \%)$ que não mostraram arrependimento, evidenciaram características antissociais, cinco (31\%) apresentaram indícios de déficit relacional e os demais $(N=4 ; 25 \%)$ demonstraram traços de déficits cognitivos.

Os dados levantados por meio das entrevistas confirmaram as informações fornecidas por outros estudos citados na revisão bibliográfica deste artigo (OMS, 2002; Bartol, 2008). Os fatores familiares, situacionais, sociais e de personalidade se mostraram presentes na história de cada um dos participantes e podem ter favorecido os comportamentos violentos.

De uma forma geral, diante da análise das variáveis do método de Rorschach, 16 adolescentes (48,5\%) se enquadraram no grupo em que sobressaem traços de psicopatia; $27,3 \%(N=9)$ apresentaram características que condizem ao grupo com déficits relacionais; e seis participantes $(18,2 \%)$ no grupo com déficits cognitivos. Duas adolescentes $(6 \%)$ não obtiveram características suficientes que as permitissem ingressar em algum dos grupos.

Oito adolescentes da amostra revelaram no método de Rorschach variáveis que os incluíram em dois grupos, ou seja, 24,2\% dos adolescentes apresentaram comorbidade entre dois perfis psicológicos. Diante dessa circunstância, os critérios para análise de qual grupo esses jovens se enquadrariam baseou-se em: 1) privilegiar os déficits cognitivos em relação aos demais grupos de variáveis conforme a estratégia de interpretação do SC. Essa condição foi estabelecida por partir do pressuposto de que a desorganização cognitiva é o que provoca o maior impacto na organização psicológica do indivíduo, que geram os transtornos psíquicos mais graves e as interpretações mais equivocadas da realidade. Essa estratégia de analisar os dados foi baseada na interpretação do Sistema Compreensivo (Exner, 2003), em que os distúrbios do pensamento são considerados inicialmente como a primeira variável-chave para a interpretação dos dados (p. 228); 2) na ausência da condição anterior, se há uma comorbidade entre os grupos de aspectos de psicopatia e déficits relacionais, predomina o primeiro grupo pelo fato da possibilidade de adolescentes que apresentam características antissociais terem maiores chances de desenvolver traços psicopáticos no futuro (Schmitt et al., 2006; Viljoen et al., 2010).

O grupo de adolescentes classificados no agrupamento de déficits cognitivos $(\mathrm{XA} \%<0,70$; WDA $\%<$ 0,$75 ; \mathrm{Xu} \% \geq 0,38 ; \mathrm{X}-\% \geq 0,40 ; P \leq 2 ; \mathrm{PTI} \geq 2 ; \mathrm{M}->$ 1; Zf $\leq 4$ e DQ+ < 4) (Exner, 2003, Exner \& Sendín, 1999) apresenta sérias dificuldades para perceber 
as coisas como a maioria das pessoas do seu meio. Esse tipo de desajustamento da percepção geram interpretações equivocadas da realidade, de si mesmo e das ações dos outros. Esse quadro geral acaba propiciando condutas afastadas do convencional, o que pode predispor o indivíduo a se comportar de modo desadaptado socialmente, como foi o caso desses jovens que cometeram um atentado contra a vida de outro.

No grupo com a prevalência de déficits relacionais, foram consideradas as variáveis do Rorschach que avaliam essa questão (CDI $\geq 5, \mathrm{COP}=0$, PHR $>$ $\mathrm{GHR}, \mathrm{EA} \leq 3)$. Foram incluídas aqui outras três variáveis: a variável que indica limitações em responder às situações que exigem uma maior complexidade emocional (Blends $\leq 2$ ), a variável que aponta uma estilo evitativo de responder às situações $(\mathrm{L} \geq 2)$ e outra que revela baixa autoestima $($ Ego $<0,37)$, o que predispõe a pessoa a se deixar influenciar pelo que os outros dizem, uma vez que se sente insegura e incapaz de acreditar em seus valores (Exner, 2003; Exner \& Sendín, 1999). Optou-se por encaixá-las aqui por entender que elas podem intervir de modo prejudicial também nas relações interpessoais.

No caso desses adolescentes, nota-se a dificuldade em lidar com as múltiplas tensões da vida cotidiana e, assim, eles acabam simplificando excessivamente suas percepções, buscando nunca se envolver profundamente nas situações (Resende, 2009; Exner \& Sedin, 1999). Esse comportamento é típico de pessoas com um campo de visão estreito, que decidem sem pensar muito, com pouco investimento emocional para não tomar consciência de circunstâncias ameaçadoras ou indesejáveis em suas vidas e que mantêm o equilíbrio devido a uma vida restrita e de esquiva. Com a simplificação excessiva da percepção, acabam por tomar atitudes de forma precipitada sem analisar a situação como um todo e, por conseguinte, acabam perdendo o controle diante das situações e agindo sem pensar. Segundo Exner e Sendín (1999), essas características podem se mostrar bastante frequentes em indivíduos com traços psicopáticos, que não refletem e atuam de forma repentina.

Gacono et al., (2008) observaram em seus estudos que a falta de habilidades sociais e a dificuldade em se posicionar no meio, de se aproximar das pessoas e manter vínculos saudáveis, contribui consideravelmente para que pessoas com esse perfil adotem comportamentos inadequados na sociedade.

Por fim, foram incluídos no grupo de Característica que revela traços de psicopatia os adolescentes que apresentaram até quatro dessas características mais proeminentes: narcisismo $(\mathrm{Fr}+\mathrm{rF}>0)$, juntamente com a falta de interesse pelo ser humano $(H \leq 1)$, ausência de indício de remorso por alguma atitude, com consequências emocionais irritativas e dolorosas $(\mathrm{SumV}=$ $0)$ e ausência de qualquer indício de maior interesse de contato físico e proximidade com o outro (SumT $=0$ ), capacidade insuficiente de vivenciar as emoções, reconhecer e expressar os seus sentimentos e participar de situações que envolvem expressões de afetos. (WSumC $\leq 1,5 / \mathrm{Afr}<0,40)$. Características essas evidenciadas nos estudos de Gacono e Meloy (1994), Gacono et al., (2008) e Souza e Resende (2012b).

A presença de reflexo no teste assinala a existência de componentes narcisistas integrados na organização da personalidade desses indivíduos. Esse elemento gera no indivíduo uma necessidade de reafirmação e confirmação exagerada da própria valia, elevando suas aspirações de importância social. Entretanto, não se pode afirmar que o reflexo é uma patologia por si só, como foi relatado por Exner e Sendín (1999), pois é necessário avaliar as demais características do indivíduo além do meio onde ele vive.

Portanto, a união das quatro variáveis num protocolo, e ainda o fato de os adolescentes terem crescido em um meio pouco generoso e nada sustentador das suas necessidades de atenção, sugere que há grandes possibilidades de promover condutas abertamente antissociais (Exner, 2003; Gacono et al., 2008). O indivíduo com essas características prima pela distância interpessoal, pela falta de vínculos significativos e pela supervalorização do seu valor pessoal, tornando-se preocupado com suas próprias necessidades em detrimento da preocupação com as necessidades dos outros, sem qualquer indício de culpa $(H \leq 1+\operatorname{Sum} T=0+$ Reflexo $>0$ e SumV $=0$ ). Infere-se, à vista disto, que há uma predisposição para explorar e manipular o outro para atingir seus próprios fins, sem remorso ou arrependimento, haja vista que essas quatro características de personalidade estão presentes no protocolo de Rorschach e, ao mesmo tempo, há a vivência prolongada de fatores sociais, situacionais e familiares de risco pra que o jovem adquira comportamentos violentos (Corredor, 2000; Gacono et al., 2008; Loving \& Russell, 2000; Olin \& Keatinge, 1998; Lowen, 1983; Garcia, 1999; Souza \& Resende, 2012b).

Duas adolescentes não apresentaram características suficientes para se incluírem em algum grupo. Uma delas participou de um latrocínio e se dizia arrependida com o ato, alegou que o fator motivacional 
foi a baixa condição financeira, pois ela acabou indo para a prostituição para conseguir sustentar o filho de um ano. Segundo os dados alcançados pela perícia criminal, a outra jovem assassinou a avó. Ela nega o ato, mas relata que ela e a avó brigavam com frequência, especialmente devido ao seu envolvimento com o tráfico. Em ambos os testes os aspectos que mais chamaram a atenção foram: características de estresse emocional, ansiedade e tensão, com traços de sentimentos de culpa e remorso (Sum Sombreados $=3$ e 4; SumV =1). Além disso, a jovem que cometeu latrocínio apresenta uma grande insatisfação própria com tendência a autocríticas negativas $($ Ego $=0,47, \mathrm{Fr}+\mathrm{rF}=0, \mathrm{SumV}=1)$. Ela revela ainda características de imaturidade emocional, podendo ter comportamentos de violentas descargas afetivas (0FC:1CF+1C). A adolescente que cometeu o ato contra a avó evidencia, além do estresse emocional, uma sobrecarga interna que tem ocasionado um estresse mental persistente e estável com ideações não deliberadas que dificultam a concentração em atividades cotidianas. Ela tende a ser mais ansiosa, irritável, impulsiva e com baixa tolerância a frustração $(\mathrm{FM}+\mathrm{m}=9 ;$ Nota $\mathrm{D}=-2 ; \operatorname{Adj} \mathrm{D}=-1)$. Todos esses aspectos podem gerar certo prejuízo da adaptação, especialmente a impulsividade e súbito descontrole emocional apresentados por elas (Exner \& Sedin, 1999; Resende, 2009; Souza \& Resende, 2012b).

\section{Considerações Finais}

A proposta deste estudo consistiu em levantar os diferentes aspectos psicológicos de adolescentes que cometeram homicídio. Com os dados alcançados, foi possível separar esses adolescentes em grupos com perfis de personalidade diferentes, para compreendermos melhor como eles pensam, sentem e se comportam. Trata-se de um estudo descritivo e exploratório. Contudo, para generalização dos dados, é exigido um número muito maior de participantes.

As percepções individuais e sociais da violência são elos cruciais na compreensão da gênese do problema (Assis et al., 2004). É importante destacar que qualquer estratégia de prevenção da criminalidade violenta deve considerar a existência de uma série de fatores que predispõem a ocorrência dos atos violentos. Os fatores sociais e familiares apresentados por esses jovens - como baixo nível socioeconômico, uso de drogas, envolvimento em outros atos infracionais, presenciar violência intrafamiliar, ser vítima de maus tratos
- apontam para uma intervenção focada no âmbito das políticas públicas voltadas especialmente para a prevenção dessas condutas inadequadas, como diminuição da desigualdade social e do desemprego, incremento da participação comunitária, valorização da educação e ênfase na ressocialização dos adolescentes (Freitas \& Ramires, 2011).

Considerando os aspectos individuais, foi possível discriminar três grupos diferentes de perfis de adolescentes que cometeram homicídio. Apenas duas adolescentes não se encaixaram em nenhum dos grupos. No caso dessas adolescentes há uma maior propensão de engajamento em tratamentos e encaminhamentos psicoterapêuticos que possam ajudá-las a evitar comportamentos inadequados ao se inserirem no meio social. Um tratamento focado especialmente na modulação dos afetos e tolerância a frustração (Souza \& Resende, 2012b).

No primeiro grupo, encontram-se os jovens com mais prejuízos cognitivos. Esse tipo de comprometimento pode gerar falhas frequentes na capacidade de antecipar as consequências de suas ações e de discriminar os limites do comportamento adequado na maioria das situações. Suas percepções imprecisas podem constituir uma fonte crônica e generalizada de dificuldade de adaptação na vida (Exner \& Sedin, 1999; Resende, 2009). A maioria das pessoas com esse grau de comprometimento cognitivo têm dificuldades para gerenciar os aspectos psicológicos básicos da vida diária sem a ajuda ou a supervisão de um psicólogo (Souza \& Resende, 2012b).

Os participantes do grupo com pouca habilidade social apresentaram uma deficiência de enfrentamento das situações do dia a dia, revelando ainda carcaterísticas de comportamentos mais evitativos e restritos ao lidar com as circunstâncias. Esses jovens podem frequentemente se beneficiar de tratamento focado na redução da ansiedade interpessoal e no treinamento de habilidades sociais, com o objetivo de focar na melhora da autoestima e na qualidade relacional (Del Prette \& Del Prette, 2001). Outra medida adequada seria apresentar a eles situações simples, familiares e objetivas, em que as responsabilidades sejam repassadas por etapas, aumentando gradativamente os objetivos a serem cumpridos, para que desenvolvam maneiras cada vez mais eficazes de lidar com as situações e adquiram mais autoestima e autoconfiança.

Nos adolescentes do último grupo, observa-se a presença de traços de personalidade típicos de pessoas que desenvolvem condutas abertamente antissociais e 
traços psicopáticos. No caso desse grupo, a literatura indica que há poucos estudos sistematizados sobre o tratamento de pessoas com essas características que possam orientar a elaboração de programas mais efetivos. Talvez o mais indicado seria uma psicoterapia em grupo, com limites firmes, com pessoas que apresentam as mesmas características. Isso poderia ser bastante desafiador para estes adolescentes que apresentam comportamentos auto e heterodestrutivos, para que possam começar a identificar suas próprias características um no outro, pois todos geralmente apresentam dificuldade de lidar com a desconfiança, fogem de intimidade, tendem a agir com desonestidade e também têm dificuldade de trabalhar com metas a longo prazo, assim como de pensar nas consequências de suas ações antes de agir (Costa \& Valerio, 2008; Gabbard, 1998; Kaplan \& Sadock, 2007).

É importante notar que as características de personalidade apontadas aqui devem ser consideradas com cautela. É bem possível que os jovens que apresentam esses mesmos traços podem não cometer homicídio. Vale lembrar que a combinação de vários fatores de risco, por certo período de tempo, especialmente durante a infância, encoraja o indivíduo ao comportamento violento.

O estudo apresenta limitações já que o ideal seria ter um grupo com controle de adolescentes que não cometeram homicídio, para se ter uma comparação dos resultados e analisar as características diferentes em adolescentes que cometeram atos infracionais graves daqueles que não cometeram esses atos. Além disto, com um aumento da amostra seria possível verificar se existem outros perfis de personalidade em adolescentes que cometeram homicídio. Pesquisas que exploram diferentes tipos de homicídio praticado por adolescentes (por exemplo, parricídio, homicídio relacionado a gangues e homicídio sexual) iriam fornecer mais informações sobre as diferentes motivações, traços e influências sobre esse fenômeno, bem como poderia oferecer informações adicionais que ajudariam na prevenção dessas condutas.

Outras limitações nos impedem de tirar conclusões mais definitivas com base nesses resultados. Em primeiro lugar, a amostra é relativamente modesta em tamanho e os dados foram coletados a partir de duas instituições. Portanto, os resultados são descritivos e referentes a essas duas instituições no momento em que os dados foram coletados. Além disso, o processo de coleta de dados não foi realizado por um pesquisador que era cego aos objetivos do estudo e ao projeto de pesquisa. Em terceiro lugar, a validade das várias medidas utilizadas também pode ser questionada, pois, neste estudo, foram utilizadas aquelas variáveis que apareceram com maior frequência nos estudos teóricos, e não necessariamente aquelas que apareceram nos sujeitos avaliados. Da mesma forma, os dados normativos para adolescentes no Brasil, especialmente na faixa etária de 15 a 17 anos ainda não foram publicados. Nesses casos, foram usadas as normas para adultos, que compreendeu alguns poucos sujeitos de 17 anos ( $2 \%$ da amostra de adultos), sendo os demais acima de 18 anos. Isso, obviamente, limita a nossa capacidade de inferir propriedades psicométricas adequadas para os nossos instrumentos a partir de estudos com amostras da população em geral.

No entanto, os resultados obtidos no presente estudo sugerem que o funcionamento psicológico desses adolescentes que cometeram homicídio é bem diverso, com demandas heterogêneas e, sendo assim, deveriam ser encaminhados para programas de reabilitação diferenciados.

\section{Referências}

Assis, S. G., Avanci, J. Q., Santos, N. C., Malaquias, J. V., \& Oliveira, R. V. (2004). Violência e representação social na adolescência no Brasil. Revista Panamericana de Salud Publica, 16(1), 43-51. Recuperado de http://www.scielosp.org/pdf/rpsp/ v16n1/22184.pdf

Assis, S. G. (1999). Traçando caminhos em uma sociedade violenta: A vida de jovens infratores e de seus irmãos não infratores. Rio de Janeiro: Editora Fiocruz.

Ayer, S. M., Williams, J. L., Tolan, P. H. \& Wilson, M. N. (2013). Predicting desistance in a high-risk sample: Examining the influence of individual and contextual factors. Journal of community Psychology, 41(4), 408-424. doi: 10.1002/jcop.21545

Bartol, C. R. (2008). Resilience and Antisocial Behavior. Em C. R. Bartol \& A. M. Bartol (Eds.). Current perspectives in forensic psychology and criminal behavior (pp. 81-96). London: Sage.

Borges, L. S., \& Alencar, H. M. D. (2006). Moralidade e homicídio: Um estudo sobre a motivação do transgressor. Psicologia: Reflexão e Crítica, 19(3), 451-459. doi: 10.1590/S0102-79722006000300014

Busch, K. G., Zagar, R. J., Grove, W. M., Hughes, J. R., Arbit, J., Bussell, R. E., \& Bartikowski, B. (2009). Looking forward in records of young adults

Psico-USF, Bragança Paulista, v. 21, n. 1, p. 73-86, jan./abr. 2016 
convicted of sexual homicide, rape, or molestation as youth: Risk for reoffending. Psychological Reports, 104(1), 155-184. Recuperado de http://www.ncbi. nlm.nih.gov/pubmed/19480213

Busch, K. G., Zagar, R. J., Hughes, J. R., Arbit, J., \& Bussell, R. E. (1990). Adolescents who kill. Journal of Clinical Psychology, 46(4), 472-485. Recuperado de http://www.ncbi.nlm.nih.gov/pubmed/2212052

Caldwell, M. F., McCormick, D. J., Umstead, D., \& Van Rybroek, G. (2007). Evidence of treatment progress and therapeutic outcomes among adolescents with psychopathic features. Criminal Justice and Behavior, 34(5), 573-586. doi: 10.1177/0093854806297511

Corff, Y. L., \& Toupin, J. (2009). Comparing persistent juvenile delinquents and normative peers with the Five-Factor Model of Personality. Journal of Research in Personality, 43, 1105-1108. doi:10.1016/j. jrp.2009.06.011

Corredor, M. (2000). Transtorno de conduta em niños: Um estudio de caso (Trabalho de especialização não publicado). Universidad Católica Andrés Bello, Caracas, Venezuela.

Costa, C. R. B. S. F, \& Assis, S. G. (2006). Fatores protetivos a adolescentes em conflito com a lei no contexto socioeducativo. Psicologia \& sociedade, 18(3), 74-81. doi: 10.1590/S0102-71822006000300011

Costa, J. B. P., \& Valerio, N. I. (2008). Transtorno de personalidade antissocial e transtornos por uso de substâncias: caracterização, comorbidades e desafios ao tratamento. Temas em psicologia, 16(1), 119-132. Recuperado de http://pepsic.bvsalud. org/scielo.php? script $=$ sci_arttext\&pid $=$ S1413-389 X2008000100010

Dam, C. V., Janssens, J. M., \& De Bruyn, E. E. J. (2005). PEN, Big Five, juvenile delinquency and criminal recidivism. Personality and Individual Differences, 39(1), 7-19. doi:10.1016/j.paid.2004.06.016

Del Prette, A., \& Del Prette, Z. A. P. (2001). Psicologia das relações interpessoais: Vivências para o trabalho em grupo. Rio de Janeiro: Vozes.

Dicataldo, F., \& Everett, M. (2008). Distinguishing juvenile homicide from violent juvenile offending. International Journal of Offender Therapy and Comparative Criminology, 52(2), 158-174. Recuperado de http://www.ncbi.nlm.nih.gov/pubmed/17671097
Durat, C., Jr. (2006). O Rorschach na avaliação psicológica de adolescentes em conflito com a lei (Dissertação de Mestrado não publicada). Programa de Pós-Graduação Stricto-Sensu em Psicologia da Universidade São Francisco, Itatiba, Brasil.

Exner, J. E., \& Sendín, C. (1999). Manual de interpretaşão do Rorschach para o sistema compreensivo. São Paulo: Casa do Psicólogo.

Exner, J. E., Jr. (2003). The Rorschach: A comprehensive system: Vol. 1. Basic foundations and principles of interpretation ( $4^{\mathrm{a}}$ ed.). New Jersey: John Wiley and Sons.

Formiga, N. S., Aguiar, M., \& Omar, A. (2008). Busca de sensação e condutas anti-sociais e delitivas em jovens. Psicologia Ciência e Profissão, 28(4), 668-681. doi: 10.1590/S1414-98932008000400002

Freitas, O., \& Ramires, J. C. (2011). Políticas públicas de prevenção e combate à criminalidade envolvendo jovens. Caminhos de Geografia, 12(37), 142-161. Recuperado de http://www.seer.ufu. br/index.php/caminhosdegeografia/article/ viewFile/16284/9140

Frick, P. J., \& White, S. F. (2008). Research Review: The importance of callous unemotional traits for developmental models of aggressive and antisocial behavior.Journalof ChildPsychologyandPsychiatry, 49(4), 359-375. doi: 10.1111/j.1469-7610.2007.01862.x

Gabbard, G. O. (1998). Psiquiatria psicodinâmica na prática clínica (A. E. Fillman, Trad.). Porto Alegre: Artes Médicas. (Original publicado em 1994).

Gacono, C. B., \& Meloy, J. R. (1994). The adolescent antisocial pattern. Em C. B. Gacono \& J. R. Meloy (Eds.), The Rorschach assessment of aggressive and psychopathic personalities (pp. 45-92). New Jersey: Lawrence Erlbaum Associates.

Gacono, C. B., Gacono, L. A., \& Evans, F. B. (2008). The Rorschach and antisocial personality disorder. Em C. B. Gacono, F. B. Evans, N. Kaser-Boyd, L. A. Gacono (Eds.), The Handbook of Forensic Rorschach Assessment (pp. 332-356) New York: Lawrence Erlbaum Associates.

Gallo, A. E, \& Williams, L. D. A. (2005). Adolescentes los conflito com a lei: Uma revisão dos fatores de risco par a conduta infracional. Psicologia: Teoria e Prática, 7(1), 81-95. Recuperado de http://pepsic. bvsalud.org/pdf/ptp/v7n1/v7n1a07.pdf 
Garcia, J. C. R. (1999). Aproximacion a la personalidad del homicida atraves del psicodiagnostico de Rorschach. Psicologia: Teoria e Prática, 1(2), 27-32. Recuperado de http://editorarevistas.mackenzie. br/index.php/ptp/article/view/1147

Gauer, J. C., Davoglio, T. R., \& Vasconcelos, S. J. L. (2012). Avaliação de traços antissociais em adolescentes: Perspectivas atuais. Em J. C. Gauer, S. J. L. Vasconcelos \& T. R. Davoglio (Eds.), Adolescentes em conflito: Violência, funcionamento antissocial e traços de psicopatia (pp. 35-51). São Paulo: Casa do Psicólogo.

Goldberg, L. R. (1992). The development of markers for the big-five factor structure. Psychological Assessment, 4, 26-42. Recuperado de http://projects.ori. org/lrg/PDFs_papers/Goldberg.Big-Five-Markers-Psych.Assess.1992.pdf

Gomide, P. I. C. (2010). Abuso, negligência e parricídio: Um estudo de caso. Temas em Psicologia, 18(1), 219230. Recuperado de http://pepsic.bvsalud.org/ $\mathrm{pdf} / \mathrm{tp} / \mathrm{v} 18 \mathrm{n} 1 / \mathrm{v} 18 \mathrm{n} 1 \mathrm{a} 18 . \mathrm{pdf}$

Howard, P. J., \& Howard, J. M. (1995). The Big Five quickstart: An introduction to the five-factor model of personality for buman resource professionals. Charlotte, NC: Centre for Applied Cognitive Studies.

Hutz, C. S., Nunes, C. H., Silveira, A. D., Serra, J., Anton, M., \& Wiekzorek, L. S. (1998). O desenvolvimento de marcadores para a avaliação da personalidade no modelo dos cinco grandes fatores. Psicologia: Reflexão e Crítica, 11, 395-411. doi: 10.1590/S0102-79721998000200015

Kaplan, H. I., \& Sadock, B. J. (2007). Compêndio de psiquiatria: Ciência do comportamento e psiquiatria clínica $\left(9^{\mathrm{a}}\right.$ ed.). Porto Alegre: Artmed.

Kimonis, E. R., Frick, P. J., Munoz, L. C., \& Aucoin, K. J. (2007). Can a laboratory measure of emotional processing enhance the statistical prediction of aggression and delinquency in detained adolescents with callous-unemotional traits? Journal of Abnormal Child Psychology, 35(5), 773-785. Recuperado de http://www.ncbi.nlm.nih.gov/pubmed/17510788

Kodato, S., \& Silva, A. D. (2000). Homicídios de adolescentes: Refletindo sobre alguns fatores associados. Psicologia: Reflexão e crítica, 13(3), 507-515. doi: 10.1590/S0102-79722000000300018

Loving, J., \& Russell, W. (2000). Selected Rorschach variables of psychopathic juvenile offenders. Journal of
Personality Assessment, 75, 126-142. Recuperado de http://www.ncbi.nlm.nih.gov/pubmed/10941705

Lowen, A. (1983). Narcisismo: Negação do verdadeiro self (A. Cabral, trad.). São Paulo: Editora Cultrix.

Manita, C. (2000). Das descobertas privadas aos crimes públicos: Evolução dos significados em trajetórias de droga-crime. Toxicodependências, 6(2), 17-31. Recuperado de https://repositorio-aberto.up.pt/ bitstream/10216/5539/2/81344.pdf

McMahon, R. J., Witkiewitz, K., \& Kotler, J. S. (2010). Predictive validity of callous unemotional traits measured in early adolescence with respect to multiple antisocial outcomes. Journal of Abnormal Psychology, 119(4), 752-763. doi: 10.1037/a0020796.

Minayo, M. C. de S., \& Souza, E. R. de (2003). Violência sob o olhar da saúde: A infrapolitica da contemporaneidade brasileira. Rio de Janeiro: Editora Fiocruz.

Monahan, K. C., Steinberg, L., \& Cauffman, E. (2009). Affiliation with antisocial peers, susceptibility to peer influence, and antisocial behavior during the transition to adulthood. Developmental Psychology, 45(6), 1520-1530. doi: 10.1037/a0017417.

Olin, J., \& Keatinge, C. (1998). Rapid psychological assessment. New York: John Wiley.

Organização Mundial De Saúde - OMS. (2002). Relatório Mundial sobre Violência e Saúde. Genebra: Organização Mundial de Saúde.

Pacheco, J., Alvarenga, P., Reppold, C., Piccinini, C. A., \& Hutz, C. S. (2005). Estabilidade do comportamento antissocial na transição da infância para a adolescência: uma perspectiva desenvolvimentista. Psicologia: reflexão e crítica, 18(1), 55-61. doi 10.1590/ S0102-79722005000100008

Paludo, S. (2011). Valores e normas sociais de jovens em conflito com a lei. Em D. D. Dell'Aglio, \& S. H. Koller (Eds.), Adolescência e juventude: Vulnerabilidade e contextos de proteção (pp. 139-161). São Paulo: Casa do Psicólogo.

Resende, A. C. (2009). Método de Rorschach: Referências essenciais. Goiânia: Dimensão.

Resende, A. C. (2011). A personalidade de adolescentes que cometeram homicídio por meio do método de Rorschach. Estudos, 38(1), 29-38. Recuperado de http://seer.ucg.br/index.php/estudos/article/ view/1913 
Resende, A. C., \& Souza, C. C. (2011). O método de Rorschach aplicado em avaliações de adolescentes que cometeram homicídio: Dados preliminares. Em VIII Encontro Nacional da Associação Brasileira de Ensino de Psicologia. Anais do VIII Encontro Nacional da Associação Brasileira de Ensino de Psicologia, Goiânia, Goiás, Brasil.

Ridenour, T. A., Marchant, G. J., \& Dean, R. S. (2001). Is the revised psychopathy checklist clinically useful for adolescents? Journal of Psychoeducational Assessment, 19(3), 227- 238. doi: 10.1177/073428290101900303

Roe-sepowitz, D. E. (2009).Comparing male and female juveniles charged with homicide child maltreatment, substance abuse, and crime details. Journal of interpersonal violence, 24(4), 601-617. doi: $10.1177 / 0886260508317201$

Romero, E. Luengo, M. A., \& Sobral, J. (2001). Personality and antisocial behavior: Study of temperamental dimensions. Personality and Individual Differences, 31, 329-348. doi:10.1016/S0191-8869(00)00139-2

Salekin, R. T. (2002). Factor-analysis of the millon adolescent clinical inventory in a juvenile offender population: Implications for treatment. Journal of Offender Rehabilitation, 34(3), 15-29. doi: 10.1300/ J076v34n03_02

Schmitt, R., Pinto, T. P., Gomes, K. M., Quevedo, J., \& Stein, A. (2006). Personalidade psicopática em uma amostra de adolescentes infratores brasileiros. Revista de Psiquiatria Clinica, 33(6), 297-303. doi: 10.1590/S0101-60832006000600002
Souza, C. C. de, \& Resende, A. C. (2012a). Transtornos psicológicos em adolescentes socioeducandos. Avaliação Psicológica, 11(1), p. 95-109. Recuperado de http://pepsic.bvsalud.org/pdf/avp/v11n1/ v11n1a10.pdf

Souza, C. C. de; \& Resende, A. C. (2012b). Diferentes perfis psicológicos de adolescentes que cometeram homicídio. Em D. M. Amparo, E. T. K. Okino, F. L. Osório, C. L. C. Hisatugo \& M. Tavares (Eds.). Métodos projetivos e avaliação psicológica: Atualizações, avancos e perspectivas (pp. 470-485). Brasília, DF: Associação Brasileira de Rorschach e Métodos Projetivos.

Viljoen, J. L., MacDougall, E. A., Gagnon, N. C., \& Douglas, K. S. (2010). Psychopathy evidence in legal proceedings involving adolescent offenders. Psychology, Public Policy, and Law, 16(3), 254-283. doi: 10.1037/a0019649

Vries, A., \& Liem, M. (2011). Recidivism of juvenile homicide offenders. Behavioral Sciences \& the Law, 29, 483-498. doi: 10.1002/bsl.984.

Zagar, R. J., Busch, K. G., Grove, W. M., Hughes, J. R., \& Arbit, J. (2009a). Looking forward and backward in records for risks among homicidal youth. Psychological Reports, 104(1), 103-127. Recuperado de http://www.ncbi.nlm.nih.gov/pubmed/19480211

Recebido em: 17/10/2014 Primeira reformulação em: 04/03/2015 Segunda reformulação em: 06/04/2015 Aprovado em: 28/04/2015 
Nota das autoras:

Apoio: Prosup/Capes

Sobre as autoras:

Carolina Cardoso de Souza é mestre em Psicologia (2013) pela PUC Goiás (bolsa PROSUP/CAPES), especialista em Avaliação Psicológica (2014), graduada em Psicologia (2010) e tem experiência na área de avaliação psicológica, atuando especialmente com Avaliação de Personalidade, Medidas da Ppsicologia e testes projetivos, como: método de Rorschach, teste de Zulliger, pirâmides coloridas de Pfister, entre outros.

E-mail:ccardosodesouza@gmail.com

Ana Cristina Resende é pós-doutoranda pela UNIFESP (bolsa pela FAPESP), doutora em Psicologia pela PUC RS (2009), com estágio de doutorado pela California School of Professional Psychology AIU San Diego, CA, mestre em Psicologia e psicóloga pela PUC Goiás (2001 e 1997), atualmente, é professora adjunta da graduação e pós-graduação stricto sensu da PUC Goiás, diretora do IGAP e atua principalmente com: Avaliação Psicológica, Personalidade, Psicopatologia, Método de Rorschach, Fundamentos e Medidas da Psicologia.

E-mail:profa.resende@gmail.com

Contato com as autoras:

Carolina Cardoso de Souza

Rua Jequitibá, Quadra 138 Lote 48 - Setor Santa Genoveva

Goiânia/Goiás

CEP: 74672-600

E-mail: ccardosodesouza@gmail.com 